\title{
On the decoupling of heavy states in higher-derivative supersymmetric field theories
}

\author{
M. Cvetič $, 1,2, *$ T. Mariz,, ,, and A. Yu. Petrov, 4 , \\ ${ }^{1}$ Department of Physics and Astronomy, University of Pennsylvania, \\ Philadelphia, PA 19104-6396, USA \\ 2 Center for Applied Mathematics and Theoretical Physics, \\ University of Maribor, SI2000 Maribor, Slovenia \\ ${ }^{3}$ Instituto de Física, Universidade Federal de Alagoas, \\ 57072-270, Maceió, Alagoas, Brazil \\ ${ }_{4}^{4}$ Departamento de Física, Universidade Federal da Paraíba, \\ Caixa Postal 5008, 58051-970, João Pessoa, Paraíba, Brazil
}

\begin{abstract}
:
We study the problem of decoupling of heavy chiral superfields in four-dimensional $N=1$ supersymmetric field theories with Lorentz-invariant and Lorentz-violating higher-derivative terms. We demonstrate that the earlier found effect of large logarithmic quantum corrections, due to heavy chiral superfields, takes place not only if the theory possesses quantum divergences, but also for essentially finite theories involving higher derivative terms, both Lorentz-invariant and Lorentz-breaking ones.
\end{abstract}

*Electronic address: cvetic@physics.upenn.edu

$\dagger$ Electronic address: tmariz@fis.ufal.br

${ }^{\ddagger}$ Electronic address: petrov@fisica.ufpb.br 


\section{INTRODUCTION}

The four-dimensional $N=1$ supersymmetric effective action with chiral superfields naturally arises as a subsector of the low-energy limit of compactified superstring theory. In this context it is important to address the impacts of massive modes, associated with the string theory scale $M_{\text {String }}$, on the effective four-dimensional action. In perturbative heterotic string theory the string scale is determined to be $M_{\text {String }} \sim 10^{-2} M_{\text {Planck }}[1]$. For perturbative Type II string theory compactifications the string scale could, in principle, be pushed all the way to the electroweak scale [2, 3]. In the effective field theory, the impacts of massive modes are subject to the decoupling theorem [4]. Namely, if one considers the theory involving light and heavy (super)fields, say, with mass $M$ which is of the order of $M_{\text {String }}$, the effective action of light fields is represented as a sum of dimension four-operators, and terms suppressed by the factors proportional to $M^{-n}$ (with $n \geq 1$ ). The latter ones decouple, as $M \rightarrow \infty$. For sectors of $N=1$ supersymmetric theory with chiral superfields, this conclusion has been verified at the tree level in [1]. When quantum corrections are taken into account, it turns to be that there are new corrections, which are not suppressed at large $M$, but instead their contribution grows logarithmically with $M[5]$. This result formally does not contradict the Appelquist-Carazzone theorem [4], as after an appropriate definition of physically measured couplings, the effective action involves light superfields only, whose quantum corrections are not suppressed [5].

The $N=1$ supersymmetric field theory considered in [5] had the following features: first, it did not not involve higher derivatives and second, at the quantum level it was divergent, so that quantum corrections depended on the normalization scale $\mu$, involving terms proportional to $\ln \frac{M^{2}}{\mu^{2}}$, which were responsible for the above-mentioned significant quantum corrections. In this paper we would like to address how the decoupling theorem manifests itself in higher-derivative (super)field theories. Note that higher-derivative terms naturally emerge in effective theories of compactified string theory, cf. [6]. In this paper, we focus on the the supersymmetric higher derivative sector with chiral superfields, and calculate quantum corrections there. We employ the superfield approach to calculate the effective potential described in great details in [7], and follow the conventions of that book. Technically, we use the methodology of a summation over cycle-like one-loop diagrams developed for superfield theories in [8] and further applied to different theories in many papers, including [5]. We consider several examples of higher derivative terms, including those that break Lorentz invariance. The case with Lorentz-violating terms is of special interest: as it was argued in [9], the presence of Lorentz-violating terms, which are of a special form $(n \cdot \partial)^{N}$, with $n_{a}$ is a space-like vector, and $N$ is a positive integer umber, allows for an implementation of higher derivatives without ghosts. In this paper, such terms are implemented in a superfield context for the first time, thus developing a methodology for Lorentz-violating extension to superfield models, earlier proposed in [10]. In the superfield formalism, these Lorenz-violating terms are added to a classical action in a manifestly supersymmetric way, 
i.e. without deformations of supersymmetry algebra or an introduction of new superfields.

Our key result is that the presence of higher derivatives in an effective action introduces large, typically logarithmic, quantum corrections, independent of the fact whether the theory is finite or divergent.

The structure of the paper is the following. In section 2, we summarize the results in $N=1$ supersymmetric theories with massive superfields without higher derivatives, where the renormalization gives rise to large logarithmic quantum corrections. In the section 3, we consider $N=1$ supersymmetric theories with different examples of higher-derivative terms, which are superficially finite, and derive how large quantum corrections arise in such theories. In section 4 we summarize the results and discuss implications.

\section{QUANTUM CORRECTIONS IN THEORIES WITHOUT HIGHER DERIVATIVES}

We start our study by considering the four-dimensional $N=1$ supersymmetric theory with chiral superfield, without higher derivatives. The simplest superfield model involving both light (massless) and heavy superfields is given by the classical action

$$
S=\int d^{8} z(\phi \bar{\phi}+\Phi \bar{\Phi})+\left(\int d^{6} z\left(\frac{1}{2}\left(M \Phi^{2}+\lambda \Phi \phi^{2}+f \phi \Phi^{2}\right)+\frac{g}{3 !} \phi^{3}\right)+\text { h.c. }\right) .
$$

Here $\phi$ is a light chiral superfield, which for the sake of simplicity, we choose it to be a massless chiral superfield. $\Phi$ is a heavy superfield with a large mass $M$. As a concrete example we choose $M$ to be of the order of $M_{\text {String }} \sim 10^{-2} M_{\text {Planck }}$ in perturbative heterotic string theory compactification, as in [1, 5]. Note that in perturbative Type II string compactification $M_{\text {String }}$ could be chosen to be many orders of magnitude smaller than $M_{\text {Planck }}$.

The above tree-level action slightly differs from the one considered in [1], and in [5], where the vertex proportional to $f$ was absent. We introduced it here to couple heavy and light superfields in a manner convenient for our study. Nevertheless, the tree-level situation is not qualitatively different from [1]. Indeed, one can find the equation of motion for $\Phi$ :

$$
-\frac{1}{4} \bar{D}^{2} \bar{\Phi}+(M+f \phi) \Phi+\frac{\lambda}{2} \phi^{2}=0
$$

so that we can write the solution for $\Phi$ via an iterative method as $\Phi=\Phi_{0}+\Phi_{1}+\ldots$, where the zero approximation is

$$
\Phi_{0}=-\frac{\lambda \phi^{2}}{2(M+f \phi)}
$$

being of first order in $\frac{1}{M}$, and for $k$-th order one has

$$
\Phi_{k+1}=\frac{1}{4} \frac{1}{M+f \phi} \bar{D}^{2} \bar{\Phi}_{k}
$$


i.e. the $k$-th order is suppressed at least as $M^{-(k+1)}$. The same situation occurred within the study of the tree-level effective action in [1].

However, the situation turns out to be much more delicate if we consider quantum corrections. Although we restrict ourselves to the one-loop order, the results are remarkable. In this case it is very easy to illustrate the origin of significant quantum corrections.

For the calculation of the one-loop corrections, we employ the loop expansion formalism. To do this, we split the superfields $\{\phi, \Phi\}$ into a sum of the background (classical) superfields $\left\{\phi_{0}, \Phi_{0}\right\}$ and the quantum ones $\left\{\phi_{q}, \Phi_{q}\right\}$, via the rule $\phi \rightarrow \phi_{0}+\phi_{q}$, and $\Phi \rightarrow \Phi_{0}+\Phi_{q}$. It is well known that within the one-loop approximation, we must keep only the second order in quantum superfields over which we should then integrate.

For the sake of simplicity, we choose that the light field $\phi$ is a purely a background one, while the heavy one $\Phi$ is a purely quantum one. (Indeed, as we already noted, the contributions that arise due to the presence of background heavy fields yield only corrections suppressed by $M^{-n}$, with $n \geq 1$, cf. [5], so, we can neglect the background $\Phi$ within the lower-order approximation.) The quadratic action of the quantum field $\Phi_{q}=\Phi$ takes the form

$$
S_{q}=\int d^{8} z \Phi \bar{\Phi}+\left(\frac{1}{2} \int d^{6} z(M+f \phi) \Phi^{2}+\text { h.c. }\right)
$$

We incorporate the mass into the background field $\Psi \equiv M+f \phi$. As a result, the propagator of $\Phi$ has the usual form [7]:

$$
<\Phi \bar{\Phi}>=-\frac{\bar{D}^{2} D^{2}}{16 \square} \delta^{8}\left(z_{1}-z_{2}\right)
$$

Therefore, the one-loop effective potential is contributed by a sum of supergraphs depicted in Fig. 1.
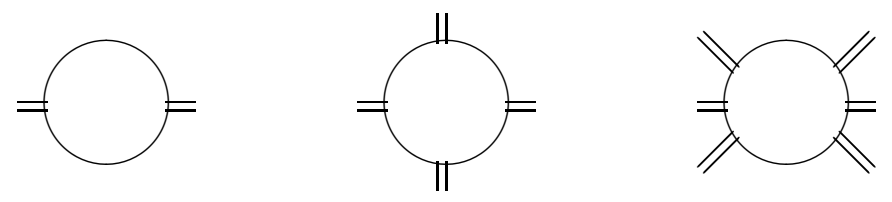

FIG. 1: The double legs denote alternating $\Psi$ and $\bar{\Psi}$ background fields. The single internal line denotes $<\Phi \bar{\Phi}>$ propagators.

We note that this set of supergraphs completely describes the one-loop effective potential in a generic chiral superfield theory whose quadratic action is

$$
S=\int d^{8} z \Phi \hat{T} \bar{\Phi}+\frac{1}{2}\left(\int d^{6} z \Psi \Phi^{2}+\text { h.c. }\right),
$$

where $\Psi$ is any background chiral superfield, and $\Phi$ is a quantum one, and $\hat{T}$ is any operator commuting with supercovariant derivatives. Actually, it must be a function of space-time 
derivatives only, being in Lorentz-invariant case a function of $\square$. In the standard case, $\hat{T}=1$.

In these diagrams, depicted in Fig. 1, the double line is for the background alternating $\Psi$ and $\bar{\Psi}$ fields. The supergraph of such structure with $2 n$ legs represents itself as a ring containing $n$ links of the form depicted in Fig. 2.

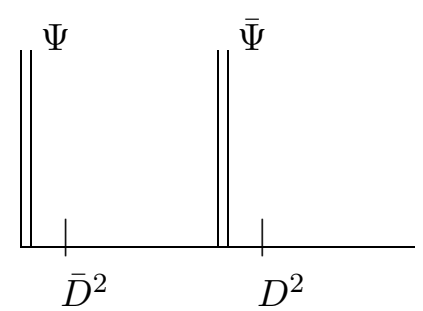

FIG. 2: The link composed by superfield propagators and background fields. Repeating of these links forms the one-loop graphs.

In this case the calculations are the same as in [8], so, we merely quote the result. After a subtraction of the divergence, we obtain the one-loop corrected Kählerian potential:

$$
K^{(1)}=-\frac{1}{32 \pi^{2}} \Psi \bar{\Psi} \ln \frac{\Psi \bar{\Psi}}{\mu^{2}} .
$$

Adding this contribution to the classical action (11), we get the following one-loop corrected low-energy effective action:

$$
\Gamma=S+\int d^{8} z K^{(1)}
$$

Note that the one-loop chiral effective potential is zero and the auxiliary fields' effective potential contributes only to higher terms in the derivative expansion, see [7]. Therefore the explicit form of (8) is

$$
\begin{aligned}
\Gamma & =\int d^{8} z\left(\phi \bar{\phi}+\Phi \bar{\Phi}-\frac{\hbar}{32 \pi^{2}}(M+f \phi)(M+f \bar{\phi}) \ln \frac{(M+f \phi)(M+f \bar{\phi})}{\mu^{2}}\right)+ \\
& +\left(\int d^{6} z\left(\frac{1}{2}\left(M \Phi^{2}+\lambda \Phi \phi^{2}+f \phi \Phi^{2}\right)+\frac{g}{3 !} \phi^{3}\right)+\text { h.c. }\right) .
\end{aligned}
$$

Here we kept the Planck constant $\hbar$ explicitly. We expand this expression in series of $M$ (remind that $M$ is large) and obtain:

$$
\begin{aligned}
\Gamma & =\int d^{8} z\left(\phi \bar{\phi}+\Phi \bar{\Phi}-\frac{\hbar}{32 \pi^{2}} f^{2} \phi \bar{\phi}\left(2+\ln \frac{M^{2}}{\mu^{2}}\right)\right)+ \\
& +\left(\int d^{6} z\left(\frac{1}{2}\left(M \Phi^{2}+\lambda \Phi \phi^{2}+f \phi \Phi^{2}\right)+\frac{g}{3 !} \phi^{3}\right)+\text { h.c. }\right)+\mathcal{O}\left(M^{-1}\right) .
\end{aligned}
$$


Then, we eliminate $\Phi$ by its equation of motion which in the lower order in $\frac{1}{M}$ yields $\Phi=$ $-\frac{\lambda \phi^{2}}{2 M}+\mathcal{O}\left(M^{-2}\right)$, cf. [5]. It is sufficient to conclude that in lower (zero) order, the low-energy effective action is

$$
\Gamma=\int d^{8} z\left(\phi \bar{\phi}-\frac{\hbar}{32 \pi^{2}} f^{2} \phi \bar{\phi}\left(2+\ln \frac{M^{2}}{\mu^{2}}\right)\right)+\left(\int d^{6} z \frac{g}{3 !} \phi^{3}+\text { h.c. }\right)+\mathcal{O}\left(M^{-1}\right) .
$$

There is a significant correction proportional to $\ln \frac{M^{2}}{\mu^{2}}$ which was a key result of [5]. As mentioned in [5], if one adds quantum fluctuations of the light field $\phi$, there would be also an additional contribution to the effective action (the Coleman-Weinberg potential): $\Gamma_{\min }=-\frac{1}{32 \pi^{2}} \int d^{8} z g^{2} \phi \bar{\phi} \ln \frac{g^{2} \phi \bar{\phi}}{\mu^{2}}$, so that the whole low-energy effective action would be the sum $\Gamma+\Gamma_{\text {min }}$. If one fixes the normalization parameter $\mu$ to be $\mu=\alpha M$, this will imply that the contribution of the one-loop action increases with growing of $M$, as the one-loop contribution is of the form $-\frac{1}{32 \pi^{2}} \int d^{8} z g^{2} \phi \bar{\phi} \ln \frac{g^{2} \phi \bar{\phi}}{M^{2}}$. We therefore conclude that neither fixing the renormalization scale $\mu$ to be of the order of $M$ nor leaving it arbitrary will avoid the appearance of large quantum corrections in the one-loop corrected effective action.

It was demonstrated in [5] that these large quantum corrections take place in a wide class of theories involving fields with very large masses. We note that suggesting that the heavy field $\Phi$ has a nontrivial background part will not essentially modify the situation since in any case the solution for $\Phi$ is proportional at least to $M^{-1}$, and hence all terms involving $\Phi$ in the effective action will be suppressed by $M^{-1}$, cf. [5]. Thus, the presence of large quantumcorrections is an universal effect in renormalizable quantum field theories with heavy fields. Note that the presence of the factors proportional to $\ln \frac{M^{2}}{\mu^{2}}$ imply that this effect occurs in renormalizable theories, where the scale $\mu^{2}$ signifies the renormalization scale.

A natural question arises whether there is a way to get large quantum correction in finite theories, where the effective action cannot depend on an arbitrary renormalization scale $\mu$. We will consider this in the subsequent section.

\section{HIGHER-DERIVATIVE THEORIES AND HEAVY STATES}

In this section, we discuss the decoupling effects of heavy states manifests due to supersymmetric higher-derivative terms. We consider the following three prototypical examples. First, we study a minimal model where derivative term is purely of the higher-derivative form, and the usual two-derivative kinetic energy term is absent; this is only a "warm-up" toy model to study general impacts in the presence of higher derivatives. Second, we study a non-minimal model involving both the higher-derivative terms, along with the standard two-derivative kinetic energy term. This is considered as a subsector of an effective theory, arising from an ultraviolet complete one, such a superstring theory. Third, we study examples of supersymmetric higher derivative terms that break Lorentz invariance. 


\section{A. Minimal case}

We start this section with a minimal case in order to give a simplest illustration how large quantum corrections can arise for higher-derivative theories. Of course, the model is only a toy example, as the heavy fields do not have a standard two-derivative kinetic energy term.

The corresponding theory is a higher-derivative $N=1$ supersymmetric model with light and heavy superfields, given by

$$
S=\int d^{8} z\left(\phi \frac{\square}{\Lambda^{2}} \bar{\phi}+\Phi \frac{\square}{\Lambda^{2}} \bar{\Phi}\right)+\left(\int d^{6} z\left(\frac{1}{2}\left(M \Phi^{2}+\lambda \Phi \phi^{2}+f \phi \Phi^{2}\right)+\frac{g}{3 !} \phi^{3}\right)+\text { h.c. }\right),
$$

which is a simple generalization of (1). A similar theory of only one chiral superfield has been studied in [11, 12]. Here, however, we introduce a mass dimension one scale parameter $\Lambda$ which enforces that the components of the chiral superfields have a correct dimension; effectively, $\Lambda$ plays a role of the energy scale at which the higher derivatives become important, cf. [13]. In string theory compactifications this scale is naturally $\Lambda=\mathcal{O}\left(M_{\text {Planck }}\right)$.

Again, to simplify the study, we suggest $\phi$ to be purely external and $\Phi$ purely quantum. (In full analogy with the previous section, we can argue that if we suggest $\Phi$ to have a nontrivial background part, the situation will not be essentially different since already the lowest approximation for $\Phi$ is proportional to $M^{-1}$.) The quadratic action of $\Phi$ is of the form:

$$
S_{q}=\int d^{8} z \Phi \frac{\square}{\Lambda^{2}} \bar{\Phi}+\left(\frac{1}{2} \int d^{6} z(M+f \phi) \Phi^{2}+\text { h.c. }\right) .
$$

This quantum action is similar to the one considered in [11]. We can introduce again the superfield $\Psi=M+f \phi$. The propagator can be obtained by a straightforward generalization of the usual Wess-Zumino case [7] and has the form

$$
<\Phi\left(z_{1}\right) \bar{\Phi}\left(z_{2}\right)>=\frac{\Lambda^{2}}{\square^{2}} \delta^{8}\left(z_{1}-z_{2}\right)
$$

with again, as in the Wess-Zumino model, the chiral vertex carries the factor $-\frac{\bar{D}^{2}}{4}$ and the antichiral one, the factor $-\frac{D^{2}}{4}$. Following [7], we can calculate the superficial degree of divergence for this theory:

$$
\omega=2-2 P-P_{c}-E_{c}
$$

where $P$ is a number of all propagators in the theory, $P_{c}$ is a number of chiral propagators, i.e., $<\Phi \Phi>$ and $<\bar{\Phi} \bar{\Phi}>$ propagators only, $E_{c}$ is a number of external chiral lines. (It is easy to show that the nonminimal theory we are considering possesses the same superficial degree of divergence, since the Wess-Zumino kinetic term yields only subleading contributions to propagators of $\Phi, \bar{\Phi}$.) 
It is clear that for any $E_{c} \geq 1$ and $P \geq 1$, one has $\omega<0$, so, the theory is ultraviolet finite. At the same time, in the usual Wess-Zumino model one has $\omega=2-P_{c}-E_{c}$, so, if the Feynman supergraph involves no chiral propagators but only $<\Phi \bar{\Phi}>$ propagators, it can yield divergent corrections to the kinetic term, while in our case there is no divergent corrections at all. We emphasize that within this study we treat $\Lambda$ as a finite parameter of the (effective) field theory, thus there is no need to introduce counterterms as $\Lambda \rightarrow \infty$.

The low-energy effective action presented by a sum over the supergraphs depicted in Fig. 1 is

$$
\Gamma_{1}=\left.\int d^{8} z \sum_{n=1}^{\infty} \frac{1}{2 n}\left[\frac{\Psi \bar{\Psi} \Lambda^{4}}{\square^{4}} \frac{\bar{D}^{2} D^{2}}{16}\right]^{n} \delta^{8}\left(z-z^{\prime}\right)\right|_{z=z^{\prime}} .
$$

This sum is analogous to that one used in the usual Wess-Zumino and SYM cases [8], with the only difference being the fact that we have $\square^{2}$ in the denominator instead of the usual $\square$. To obtain the low-energy (Kählerian) contribution to the effective action, we disregard all terms where derivatives act on background fields. In order to sum contributions, we use the property of the projection operator $\left(\frac{\bar{D}^{2} D^{2}}{16 \square}\right)^{n}=\frac{\bar{D}^{2} D^{2}}{16 \square}$. Subsequently, we employ "the shrinking of the loop to a point" via the well-known identity $\left.\frac{\bar{D}^{2} D^{2}}{16} \delta^{8}\left(z-z^{\prime}\right)\right|_{z=z^{\prime}}=1$, and finally, employ the sum $\sum_{n=1}^{\infty} a^{n}=-\ln (1-a)$. We arrive at the following result for the Kählerian effective potential, analogous to that in [11]:

$$
K^{(1)}=\frac{1}{2} \int \frac{d^{4} k}{(2 \pi)^{4}} \frac{1}{k^{2}} \ln \left[1+\frac{|\Psi|^{2} \Lambda^{4}}{k^{6}}\right],
$$

which, by removing the field independent part, takes the form

$$
K^{(1)}=\frac{1}{2} \int \frac{d^{4} k}{(2 \pi)^{4}} \frac{1}{k^{2}} \ln \left[\frac{k^{6}}{\Lambda^{4}}+|\Psi|^{2}\right]
$$

which yields

$$
K^{(1)}=\frac{c_{0}}{32 \pi^{2}}\left(\Psi \bar{\Psi} \Lambda^{4}\right)^{1 / 3}
$$

where $c_{0}$ is a finite dimensionless constant whose value can be found in [11]. We can again expand the low-energy effective action in series of $M$ (recall that $\Psi=M+f \phi$ ). As a result, we obtain

$$
K^{(1)}=\frac{c_{0}}{288 \pi^{2}} \frac{\Lambda^{4 / 3}}{M^{4 / 3}} f^{2} \phi \bar{\phi}+\mathcal{O}\left(\frac{\Lambda^{4 / 3}}{M^{7 / 3}}\right) .
$$

It is clear that after the rescaling $\phi\left(1+\frac{c_{0}}{288 \pi^{2}} \frac{\Lambda^{4 / 3}}{M^{4 / 3}} f^{2}\right)^{1 / 2} \rightarrow \phi$, together with the rescalings of constant parameters, the one-loop corrected effective action $\Gamma=S+\int d^{8} z K^{(1)}$ reproduces the classical action of the theory involving light superfields only. Thus, the decoupling theorem is formally satisfied. However, the key point in interpreting this result relies on 
the magnitude of the scale $\Lambda$. We see that if $\Lambda \ll M$, the perturbative contribution to the effective action is strongly suppressed, as $\left(\frac{\Lambda}{M}\right)^{-4 / 3}$. However, for $\Lambda \simeq M$, when there is only one energy scale in the theory, the quantum correction becomes compatible with the tree-level effective action. The case $\Lambda>M$ has a natural occurrence in string theory, where $\Lambda=\mathcal{O}\left(M_{\text {Planck }}\right)$ and $M<M_{\text {Planck }}$, e.g., in perturbative heterotic string theory $\left.M \simeq M_{\text {String }}=10^{-2} M_{\text {Planck }}\right)$. In this case quantum contributions begin to dominate.

We also note that the mechanism of large quantum corrections described in section 2 cannot be applied to the case with finite quantum contributions, discussed in this section, since now quantum corrections do not depend on the arbitrary normalization parameter $\mu$ which can arise only as a consequence of subtractions of divergences. In the higher-derivative field theory models, instead of $\mu$, there is another natural energy scale $\Lambda$, which describes a characteristic energy scale at which the higher derivatives become important. However, despite of essentially different mechanisms for the quantum corrections in divergent and higher-derivative finite theories, the general structure of quantum corrections turns out to be rather similar in both cases; the logarithmic quantum corrections, proportional to $\mu$, in renormalizable theories, and to $\Lambda$, in finite higher derivative theories, is very analogous.

We can generalize these studies to a generic theory whose action of quantum fields is given by (6), instead of (13). In this case, $\hat{T}$ is a generic scalar operator commuting with supercovariant derivatives. In particular, we can have the case $\hat{T}=1$ which corresponds to the usual Wess-Zumino model. Indeed, we would have the same background fields $\Psi$ and $\bar{\Psi}$, but our propagator $<\Phi \bar{\Phi}>$ will be of the form:

$$
<\Phi\left(z_{1}\right) \bar{\Phi}\left(z_{2}\right)>=\frac{1}{\hat{T} \square} \delta^{8}\left(z_{1}-z_{2}\right)
$$

so, we can adapt the results given by (16,17) and find

$$
K^{(1)}=\frac{1}{2} \int \frac{d^{4} k}{(2 \pi)^{4}} \frac{1}{k^{2}} \ln \left[T^{2}(k) k^{2}+|\Psi|^{2}\right]
$$

where $T(k)$ is a Fourier transform of the operator $\hat{T}$. In the simplest case $T(k)=\frac{k^{2 n}}{\Lambda^{2 n}}(n \geq 1)$, where $\Lambda$ again plays a role of an energy scale at which the higher derivatives dominate, it is easy to find this integral. The result is

$$
K^{(1)}=\frac{1}{32 \pi^{2}} \Gamma\left(\frac{1}{2 n+1}\right) \Gamma\left(\frac{2 n}{2 n+1}\right)\left(\frac{|\Psi|^{2}}{\Lambda^{2}}\right)^{\frac{1}{2 n+1}} .
$$

Again, defining $\Psi=M+\lambda \phi$ and expanding this expression as a power series in $\lambda$, we obtain

$$
K^{(1)}=k_{0}\left(\Lambda^{4 n} M^{2}\right)^{\frac{1}{2 n+1}}\left[\frac{\lambda^{2} \phi \bar{\phi}}{M^{2}}+\mathcal{O}\left(M^{-3}\right)\right]
$$

where $k_{0}$ is a purely numerical constant which does not depend on any physical scale. Therefore the scale of this expression is completely characterized by $\left(\frac{\Lambda}{M}\right)^{4 n /(2 n+1)}$. 
If we take $\Lambda \simeq M$, namely if the theory involves only one characteristic energy scale, we have

$$
K^{(1)}=\frac{k_{0}}{(2 n+1)^{2}} \lambda^{2} \phi \bar{\phi}+\mathcal{O}\left(M^{-1}\right) .
$$

Then, the quantum correction caused by coupling of a light superfield $\phi$ with heavy ones is not suppressed, as in the previous example, although we again can argue that this result is formally consistent with the decoupling theorem. And if we suggest that $\Lambda \gg M$, as e.g. in the above-mentioned case, when $M \simeq M_{\text {String }} \simeq 10^{-2} \Lambda$, this correction begin to dominate. Namely, for $\Lambda=\gamma M$, with $\gamma \gg 1$, we have

$$
K^{(1)}=\gamma^{\frac{4 n}{2 n+1}} \frac{k_{0}}{(2 n+1)^{2}} \lambda^{2} \phi \bar{\phi}+\mathcal{O}\left(M^{-1}\right) \text {. }
$$

Thus, the quantum correction is large since $\gamma^{\frac{4 n}{2 n+1}} \simeq 10^{4}$.

\section{B. Nonmimimal case}

Another example of the higher-derivative superfield theory, that includes the standard kinetic energy terms, has been discussed in [11, 12]. The action is of the form:

$$
\begin{aligned}
S & =\int d^{8} z\left(\phi \bar{\phi}+\Phi\left(1+\frac{\square}{\Lambda^{2}}\right) \bar{\Phi}\right)+ \\
& +\left(\int d^{6} z\left(\frac{1}{2}\left(M \Phi^{2}+\lambda \Phi \phi^{2}+f \phi \Phi^{2}\right)+\frac{g}{3 !} \phi^{3}\right)+\text { h.c. }\right) .
\end{aligned}
$$

For simplicity we take $\phi$ chiral superfield to be massless. Again, we choose $\phi$ to be a purely external one, and $\Phi$ to be a purely internal one. (For the sake of simplicity we do not introduce higher derivatives for the light superfields. In the effective theory of light fields, only, these terms are suppressed by $\mathcal{O}\left(\Lambda^{-1}\right)$ and decouple.) We carry out a summation over the supergraphs depicted in Fig. 1. The result, after the Wick rotation, is given by

$$
K^{(1)}=\frac{1}{2} \int \frac{d^{4} k}{(2 \pi)^{4}} \frac{1}{k^{2}} \ln \left[k^{2}\left(1+\frac{k^{2}}{\Lambda^{2}}\right)^{2}+|\Psi|^{2}\right] .
$$

The explicit result for this expression is cumbersome. Nevertheless, we can proceed with this integral in some characteristic cases.

We proceed by considering the object $J=\frac{d K^{(1)}}{d(\Psi \bar{\Psi})}$. A replacement $d^{4} k \rightarrow \pi^{2} t d t$, with $t=k^{2}$, yields

$$
J=\frac{1}{32 \pi^{2}} \int_{0}^{\infty} d t \frac{\Lambda^{4}}{t\left(t+\Lambda^{2}\right)^{2}+\Lambda^{4}|\Psi|^{2}}=\frac{1}{32 \pi^{2}} \int_{0}^{\infty} d t \frac{\Lambda^{4}}{(t+A)(t+B)(t+C)},
$$

where $A, B, C$ are three roots of the denominator, taken with opposite signs, i.e., $t\left(t+\Lambda^{2}\right)^{2}+$ $\Lambda^{4}|\Psi|^{2}=(t+A)(t+B)(t+C)$. Then, we can write

$$
\frac{1}{t\left(t+\Lambda^{2}\right)^{2}+\Lambda^{4}|\Psi|^{2}}=\frac{1}{Q}\left[\frac{B-C}{t+A}+\frac{C-A}{t+B}+\frac{A-B}{t+C}\right],
$$


where $Q=A B(A-B)+B C(B-C)+C A(C-A)$. It is clear therefore that at least one of the numbers $A-B, B-C, C-A$ will be negative, and hence at least one of the residua of the propagator (301) will be negative. Thus the action (27) unavoidably involves ghosts.

We should however note that a fundamental, ultraviolet complete theory, such as string theory, should be ghost-free. Therefore, we can treat our result in the following manners. First, one can suppose that the specific higher-derivative terms also represent themselves as contributions in the effective theory where the higher derivatives arise as a consequence of an integration over additional matter fields as it occurs, e.g., in gravity theories coupled to additional matter fields [14]. Second, we can treat higher-derivative terms as a next approximation in a derivative expansion of a fundamental non-local theory where the ghosts are avoided [15]. We note that if we abandon the Lorentz invariance, the higher derivatives can be introduced in a unitary manner through appropriate contractions with Lorentzbreaking vectors or tensors [9]. We consider this situation later.

The straightforward integration of (29) allows one to obtain the explicit result in terms of roots $A, B, C$ :

$$
J=\frac{\Lambda^{4}}{16 \pi^{2} Q}\left[A \ln \frac{B}{C}+\ln \frac{C}{A}+C \ln \frac{A}{B}\right] .
$$

In principle, one can use here the explicit expressions for $A, B, C$. They are given in the Appendix, along with their explicit asymptotic behaviour in $\frac{|\Psi|^{2}}{\Lambda^{2}} \ll 1$ and $\frac{|\Psi|^{2}}{\Lambda^{2}} \gg 1$ regimes. The explicit form of (29) in these regimes can be also analysed directly, by introducing the dimensionless quantity $R^{2}=\frac{|\Psi|^{2}}{\Lambda^{2}}$, i.e., by writing (29) as

$$
J=\frac{1}{32 \pi^{2}} \int_{0}^{\infty} d u \frac{1}{u(u+1)^{2}+R^{2}},
$$

and find the asymptotic behaviour of this integral in $R \ll 1$ and $R \gg 1$ regimes. The case $R \ll 1$ effectively corresponds to $M \ll \Lambda$ and results in the expansion:

$$
\left.J\right|_{R \rightarrow 0}=-\frac{1}{32 \pi^{2}}\left[\ln R^{2}+1+R^{2}\left(4 \ln R^{2}+\frac{19}{3}\right)\right]+\mathcal{O}\left(R^{3}\right) .
$$

The case $R \gg 1$ corresponds to $M \gg \Lambda$ and results in

$$
\left.J\right|_{R \rightarrow \infty}=-\frac{1}{96 \pi^{2}} \frac{\ln R^{2}}{R^{4 / 3}}+\mathcal{O}\left(R^{-2}\right)
$$

Thus, by integrating these expressions, respectively, we obtain

$$
\begin{aligned}
K_{R \rightarrow 0}^{(1)} & =-\frac{1}{32 \pi^{2}}\left(|\Psi|^{2} \ln \frac{|\Psi|^{2}}{\Lambda^{2}}+2 \frac{|\Psi|^{4}}{\Lambda^{2}} \ln \frac{|\Psi|^{2}}{\Lambda^{2}}+\frac{13}{6} \frac{|\Psi|^{4}}{\Lambda^{2}}\right)+\cdots ; \\
K_{R \rightarrow \infty}^{(1)} & =-\frac{1}{32 \pi^{2}}|\Psi|^{2 / 3} \Lambda^{4 / 3}\left(\ln \frac{|\Psi|^{2}}{\Lambda^{2}}+\frac{3}{2}\right)+\cdots,
\end{aligned}
$$

where again $\Psi=M+f \phi$. Further expansion of the above expressions in terms of the small field $\phi \ll M$, and disregarding field independent terms (whose contribution to the effective 
action vanishes because of properties of the integral over Grassmannian variables), yields the following expressions:

$$
\begin{aligned}
& K_{M \ll \Lambda}^{(1)}=-\frac{1}{32 \pi^{2}} f^{2} \phi \bar{\phi} \ln \frac{M^{2}}{\Lambda^{2}}+\cdots \\
& K_{M \gg \Lambda}^{(1)}=-\frac{1}{288 \pi^{2}}\left(\frac{\Lambda}{M}\right)^{4 / 3} f^{2} \phi \bar{\phi} \ln \frac{M^{2}}{\Lambda^{2}}+\cdots .
\end{aligned}
$$

Note, that in the second case $M \gg \Lambda$ the correction is suppressed. It is however the first case $M \ll \Lambda$ that one encounters in typical string theory compactifications. In particular, in perturbative heterotic string compactification $\Lambda=\mathcal{O}\left(M_{\text {Planck }}\right)$ and $M=\mathcal{O}\left(M_{\text {String }}\right)$, where $M_{\text {String }}=10^{-2} M_{\text {Planck }}$. Note that in this case there are large logarithmic corrections.

\section{Lorentz-breaking case}

In this subsection we address the study of Lorentz violating terms due to heavy superfields. As a first step we introduce supersymmetric higher derivative terms that break Lorentz invariance. For specific choices of the Lorentz breaking vector in the higherderivative terms, as proposed in [16], the theory can still maintain unitarity. As a prototype, we consider the following extension of the Wess-Zumino model:

$$
\begin{aligned}
S & =\int d^{8} z\left[\bar{\Phi}\left(1-\frac{1}{\Lambda^{2}}(n \cdot \partial)^{2}\right) \Phi+\bar{\phi} \phi\right]+ \\
& +\left(\int d^{6} z\left(\frac{1}{2}\left(M \Phi^{2}+\lambda \Phi \phi^{2}+f \phi \Phi^{2}\right)+\frac{g}{3 !} \phi^{3}\right)+\text { h.c. }\right) .
\end{aligned}
$$

Here, $n_{a}$ is a dimensionless Lorentz-breaking vector which in principle can be chosen to be space-like $\left(n_{a} n^{a}=1\right)$, to avoid higher time derivatives which spoil the unitarity. (In reality, we do all calculations in the Euclidean space, and hence this problem is avoided). This is a Myers-Pospelov-like extension to the case of the Wess-Zumino model. Here we introduced $(n \cdot \partial)^{2}$ to simplify the integration. We note that the introduction of the higher-derivative Lorentz-breaking terms in this manner, i.e. through terms $(n \cdot \partial)^{N}$ with different values of $N$, was discussed in [16].

Again, we consider the same type of graphs as in Fig. 1. We repeat the calculations, given by (16 -17), along the lines discussed earlier. Actually, the only difference is in replacement of $\frac{\Lambda^{2}}{\square^{2}}$ by $\left[\square\left(1-\frac{1}{\Lambda^{2}}(n \cdot \partial)^{2}\right)\right]^{-1}$. Repeating the summation, we arrive at

$$
K^{(1)}=\frac{1}{2} \int \frac{d^{4} k}{(2 \pi)^{4}} \frac{1}{k^{2}} \ln \left[k^{2}\left(1+\frac{(n \cdot k)^{2}}{\Lambda^{2}}\right)^{2}+|\Psi|^{2}\right] .
$$

We make a replacement $k_{a} k_{b}=\frac{1}{4} \eta_{a b} k^{2}$, which is valid within any integral over momenta of the form $\int \frac{d^{d} k}{(2 \pi)^{d}} f(k) k_{a_{1}} k_{a_{2}} \ldots k_{2 m}$. For example, in the simplest case $m=1$, with arbitrary values of $d$ and $N$, one has

$$
\int \frac{d^{d} k}{(2 \pi)^{d}} \frac{k_{a} k_{b}}{\left(k^{2}+M^{2}\right)^{N}}=\frac{1}{d} \eta_{a b} \int \frac{d^{d} k}{(2 \pi)^{d}} \frac{k^{2}}{\left(k^{2}+M^{2}\right)^{N}}=\frac{1}{2(4 \pi)^{d / 2}}\left(M^{2}\right)^{d / 2+1-N} \frac{\Gamma(N-1-d / 2)}{\Gamma(N)} ;
$$


and for larger even $m$ this formula can be naturally generalized. (We note that since the effective potential is a scalar, the Lorentz-breaking vector $n_{a}$ can enter only through a contraction $n^{a} n_{a}$ which is equal to 1 ; moreover, since our effective potential is finite, it has no singularities, and therefore its behaviour will not be modified qualitatively by changing the vector $n_{a}$.)

We proceed, as in the previous section, by calculating the asymptotic form of the exact integrals in $|\Psi|^{2} \ll \Lambda^{2}$ (effectively, $M \ll \Lambda$ ) and in $|\Psi|^{2} \gg \Lambda^{2}$ (effectively, $M \gg \Lambda$ ) regime. The result is: for $|\Psi|^{2} \ll \Lambda^{2}$,

$$
K^{(1)}=-\frac{13 \Psi^{4}}{768 \pi^{2} \Lambda^{2}}-\frac{\Psi^{2} \ln \left(\frac{\Psi^{2}}{4 \Lambda^{2}}\right)}{32 \pi^{2}}-\frac{\Psi^{4} \ln \left(\frac{\Psi^{2}}{4 \Lambda^{2}}\right)}{64 \pi^{2} \Lambda^{2}}+\cdots,
$$

and for $|\Psi|^{2} \gg \Lambda^{2}$

$$
K^{(1)}=\frac{\Psi^{2}\left(\frac{\Lambda^{2}}{\Psi^{2}}\right)^{2 / 3}}{42^{2 / 3} \sqrt{3} \pi}-\frac{\Lambda^{2} \ln \left(\frac{\Psi^{2}}{4 \Lambda^{2}}\right)}{12 \pi^{2}}+\cdots .
$$

Expanding the above expressions in power series for the small $\phi$, we have

$$
K_{M \ll \Lambda}^{(1)}=-\frac{\lambda^{2} \phi \bar{\phi}}{32 \pi^{2}}\left[3+\ln \frac{M^{2}}{4 \Lambda^{2}}\right]+\mathcal{O}\left(\frac{M}{\Lambda}, \frac{1}{M}\right)+\cdots
$$

and

$$
K_{M \gg \Lambda}^{(1)}=\left(\frac{\Lambda^{2}}{M^{2}}\right)^{2 / 3} \frac{1}{2^{2 / 3} 3^{5 / 2} \pi} \lambda^{2} \phi \bar{\phi}+\mathcal{O}\left(\frac{\Lambda}{M}, \frac{1}{M}\right)+\cdots,
$$

respectively. We see again that in the second case corrections are suppressed. It is however the first case with $\Lambda \gg M$ that one encounters in the string theory compactification. In this case the corrections have logarithmic enhancement.

It is instructive to compare our results with another type of Lorentz-violating supersymmetric theories, discussed in [10], where the classical action of the form

$$
S=\int d^{8} z \Phi\left(1+\rho \Delta^{z-1}\right) \bar{\Phi}+\left(\int d^{6} z W(\Phi)+\text { h.c. }\right)
$$

was considered, with $W(\Phi)=\frac{m}{2} \Phi^{2}+\frac{\lambda}{3 !} \Phi^{3}$. There, for the same $\Psi=m+\lambda \phi$ (where, however, the mass $m$ was not enforced to be large as in our case), the result was

$$
K^{(1)}=c \rho^{-1 / z}(\Psi \bar{\Psi})^{1 / z},
$$

where $z$ is a critical exponent, $\rho$ is a small constant whose mass dimension is $-2(z-1)$, and $c$ is a some number of the order of 1 . We can modify this theory within the framework of our approach, so that the action would be

$$
\begin{aligned}
S & =\int d^{8} z\left(\phi \bar{\phi}+\Phi\left(1+\rho \Delta^{z-1}\right) \bar{\Phi}\right)+ \\
& +\left(\int d^{6} z\left(\frac{1}{2}\left(M \Phi^{2}+\lambda \Phi \phi^{2}+f \phi \Phi^{2}\right)+\frac{g}{3 !} \phi^{3}\right)+\text { h.c. }\right) .
\end{aligned}
$$


In this case, one has $\Psi=M+f \phi$. Repeating the calculations, and introducing a cut-off scale $\Lambda$ so that we can estimate $\rho=\frac{\alpha}{\Lambda^{2 z-2}}$ by dimensional analysis, with $\alpha$ being a number of the order of 1 , we arrive at the result (44), which, for our choice of $\Psi$, can be expanded as

$$
K^{(1)}=c \frac{\alpha^{-1 / z}}{z^{2}}\left(\frac{M}{\Lambda}\right)^{\frac{2}{z}-2} f^{2} \phi \bar{\phi}+\cdots
$$

where dots are for higher orders in expansion in $M^{-1}$. (All the dependence on $\Lambda$ is concentrated in the factor $\left(\frac{M}{\Lambda}\right)^{\frac{2}{z}-2}$, so, dots are for essentially smaller terms.) We see that for $\Lambda$ and $M$ of the same order, this correction is not suppressed. Moreover, since typically $z>1$, and $\frac{2}{z}-2<0$, we see that for $M<\Lambda$ (which is a natural regime as already argued) the low-energy effective action increases as $M$ grows. Moreover, in this case the factor $\left(\frac{M}{\Lambda}\right)^{\frac{2}{z}-2}$ is large, tending to $10^{4}$ for a large $z$.

\section{SUMMARY}

In this paper we discussed the problem of decoupling of heavy states in four-dimensional $N=1$ supersymmetric field theory sector with light and heavy chiral superfields. We encountered an essential difference between the renormalizable theories, exhibiting divergent quantum contributions, and effective theories with higher derivative terms which have finite quantum corrections. In renormalizable quantum field theories, after renormalization, quantum corrections at the $L$-loop level one can have contributions proportional to $|\phi|^{2}\left(\ln \frac{|\phi|^{2}}{\mu^{2}}\right)^{L}$, where, $\mu$ is the renormalization scale. Since the only dimensional scale in the theory is a large mass $M$, it is natural to fix $\mu=M$, so, one will have significant contributions which, moreover, increase as $M$ grows. Of course, by changing the $\mu$ to be a low energy scale, this would then result in renormalized couplings, in front of such terms, which are defined at a low energy scale $\mu$.

In effective theories with higher derivative terms the quantum corrections are finite and thus do not involve the renormalization scale. However, if one considers higher-derivative extensions, one introduces the scale $\Lambda$ characterizing the energy at which the higher-derivative terms become important [13]. As we have shown, if this scale is large enough, i.e. of the order of the mass of the heavy superfield, the quantum corrections due to these terms also become significant. Therefore, we found that instead of the mechanism based on the renormalization the effects of higher derivative terms result in large finite quantum corrections. This effect is realized for both Lorentz-invariant and Lorentz-breaking higher derivative examples. In principle, this result can be formally understood if we treat higher-derivative terms as a type of a higher-derivative "regularization" that ensures finiteness of quantum corrections. It is therefore clear that in the absence of the regularization the one-loop effective action will diverge. However, within our approach the higher-derivative terms are treated not as a regularization but are a fundamental ingredient of the effective theory. Therefore, we have 
large quantum corrections which, following [9], can be interpreted as a sign of a fine-tuning in a corresponding effective theory of light fields only. Furthermore, for an example of an effective theory of the perturbative heterotic string compactification, $M \simeq 10^{-2} \Lambda$, and thus large quantum corrections, proportional to $\ln \left(\frac{M^{2}}{\Lambda^{2}}\right)$ are unavoidable. These results also imply, that theories with heavy superfields, both those with higher derivatives and those without them, possess quantum corrections that can significantly modify the low-energy effective theory of light fields. We also expect that the presence of the fields with a large mass could have cosmological impact since the presence of large quantum corrections would strongly modify the observable values of physical variables. Also, since the galileon models naturally involve higher derivatives [17], it is natural to expect that studies of the higher-derivative field theory models, in particular, the those with finite quantum corrections, can be relevant within the context of galileon cosmology.

A generalization of this study to would involve higher derivative couplings of chiral superfields to gauge superfields. However, up to now, the only models studied in this manner involve higher derivatives in the gauge sector [18]. Another direction for future studies should involve a more general and systematic study of Lorentz-breaking supersymmetric theories, in particular more generic models for chiral matter, e.g., Lorentz-breaking extensions of models discussed in [5], as well as superfield analogues of the models discussed in [16].

\section{Acknowledgments}

This work was partially supported by Conselho Nacional de Desenvolvimento Científico e Tecnológico (CNPq). A.Yu.P. has been supported by the CNPq project No. 303438-2012/6. M.C. is supported in part by the DOE (HEP) Award de-sc0013528, the Fay R. and Eugene L. Langberg Endowed Chair (M.C.) and the Slovenian Research Agency (ARRS). M.C. and A.Yu.P. would like to thank the organizers of the workshop: "Theoretical Frontiers in Black Holes and Cosmology," in Natal, Brazil, where the work was initiated, for hospitality.

\section{APPENDIX}

Here we give explicit expressions for the roots $A, B, C$ of the denominator of (29)). Using the Cardano formula, we can write 


$$
\begin{aligned}
& A=\frac{1}{3}\left(2 \Lambda^{2}+Q+\bar{Q}\right), \\
& B=\frac{1}{3}\left(2 \Lambda^{2}+\left(\frac{-1+i \sqrt{3}}{2}\right) Q+\left(\frac{-1-i \sqrt{3}}{2}\right) \bar{Q}\right), \\
& C=\frac{1}{3}\left(2 \Lambda^{2}+\left(\frac{-1-i \sqrt{3}}{2}\right) Q+\left(\frac{-1+i \sqrt{3}}{2}\right) \bar{Q}\right),
\end{aligned}
$$

where

$$
\begin{aligned}
& Q=\sqrt[3]{\frac{27 \Lambda^{4}|\Psi|^{2}-2 \Lambda^{6}+\sqrt{729 \Lambda^{8}|\Psi|^{4}-108 \Lambda^{10}|\Psi|^{2}}}{2}}, \\
& \bar{Q}=\sqrt[3]{\frac{27 \Lambda^{4}|\Psi|^{2}-2 \Lambda^{6}-\sqrt{729 \Lambda^{8}|\Psi|^{4}-108 \Lambda^{10}|\Psi|^{2}}}{2}} .
\end{aligned}
$$

It remains only to find these roots in the approximation: $\Lambda^{2} \ll|\Psi|^{2}$ (i.e. $R \equiv \frac{|\Psi|}{\Lambda} \gg 1$ ) and for $\Lambda^{2} \gg|\Psi|^{2}$ (i.e. $R \ll 1$ ). It is more convenient to consider the dimensionless objects $\tilde{A} \equiv \frac{A}{\Lambda^{2}}, \tilde{B} \equiv \frac{B}{\Lambda^{2}}, \tilde{C} \equiv \frac{C}{\Lambda^{2}}$. Actually, $\tilde{A}, \tilde{B}, \tilde{C}$ are the roots of the denominator of the Eq. (32) multiplied by -1 . (This factor is needed to return to the form of the roots corresponding to the Minkowski space.)

For $R \ll 1$, we find

$$
\begin{aligned}
\tilde{A} & =\mathcal{O}\left(R^{2}\right) ; \\
\tilde{B} & =1+\mathcal{O}(R) \\
\tilde{C} & =1+\mathcal{O}(R) .
\end{aligned}
$$

For $R \gg 1$, we have

$$
\begin{aligned}
& \tilde{A}=R^{2 / 3}+\frac{2}{3}+\mathcal{O}\left(R^{-1 / 3}\right) \\
& \tilde{B}=R^{2 / 3}+\frac{2}{3}+\mathcal{O}\left(R^{-1 / 3}\right) \\
& \tilde{C}=R^{2 / 3}+\frac{2}{3}+\mathcal{O}\left(R^{-1 / 3}\right) .
\end{aligned}
$$

We see that the roots are positive, so, there are no tachyons in the theory, however, as we already mentioned, the ghosts are unavoidable.

[1] M. Cvetič, L. Everett, J. Wang, Nucl. Phys. B538, 52 (1999), hep-ph/9807321.

[2] I. Antoniadis, Phys. Lett. B246, 377 (1990). 
[3] I. Antoniadis, N. Arkani-Hamed, S. Dimopoulos, G. R. Dvali, Phys. Lett. B436, 257 (1998), hep-ph/9804398.

[4] T. Appelquist, J. Carazzone, Phys. Rev. D11, 2856 (1975).

[5] I. L. Buchbinder, M. Cvetič, A. Yu. Petrov, Mod. Phys. Lett. A15, 783 (2000), hep-th/9903243; Nucl. Phys. B571, 358 (2000), hep-th/9906141.

[6] D. Fliegner, M. G. Schmidt, C. Schubert, Z. Phys. C64, 111 (1994), hep-ph/9401221; D. Fliegner, P. Haberl, M. G. Schmidt, C. Schubert, Ann. Phys. 264, 51 (1998), hep-th/9707189, A. Castro, J. L. Davis, P. Kraus, F. Arsen, JHEP 0706, 007 (2007), hep-th/0703087.

[7] I. L. Buchbinder, S. M. Kuzenko. Ideas and Methods of Supersymmetry and Supergravity. IOP Publishing, Bristol and Philadelphia, 1998.

[8] B. de Wit, M. T. Grisaru, M. Roček, Phys. Lett. B 374, 297 (1996), hep-th/9601115 A. Pickering, P. West, Phys. Lett. B 383, 54 (1996), hep-th/9604147; M. T. Grisaru, M. Rocek, R. von Unge, Phys. Lett. B 383, 415 (1996), hep-th/9605149.

[9] C. M. Reyes, Phys. Rev. D87, 125028 (2013), arXiv: 1307.5340; M. Maniatis, C. M. Reyes, Phys. Rev. D89, 056009 (2014), arXiv: 1401.3752.

[10] M. Gomes, J. R. Nascimento, A. Yu. Petrov, A. J. da Silva, Phys. Rev. D90, 125022 (2014), arXiv: 1408.6499 .

[11] M. Gomes, J. R. Nascimento, A. Yu. Petrov, A. J. da Silva, Phys. Lett. B682, 229 (2009), arXiv: 0908.0900.

[12] I. Antoniadis, E. Dudas, D. M. Ghilencea, JHEP 0803, 045 (2008), arXiv: 0708.0383.

[13] M. Fontanini, M. Trodden, Phys. Rev. D83, 103518 (2011), arXiv: 1102.4357.

[14] I. Antoniadis, E. Mottola, Phys. Rev. D45, 2013 (1992).

[15] L. Modesto, Phys. Rev. D86, 044005 (2012), arXiv: 1107.2403.

[16] R. Myers, M. Pospelov, Phys. Rev. Lett. 90, 211601 (2003), hep-ph/0301124.

[17] A. Nicolis, R. Rattazzi, E. Tricherini, Phys. Rev. D79, 064036 (2009), arXiv: 0811.2197.

[18] F. S. Gama, M. Gomes, J. R. Nascimento, A. Yu. Petrov, A. J. da Silva, Phys. Rev. D84, 045001 (2011), arXiv: 1101.0724. 